\title{
In Vivo Measurement of Vesicular Monoamine Transporter Type 2 Density in Parkinson Disease with ${ }^{18}$ F-AV-133
}

Nobuyuki Okamura ${ }^{1,2}$, Victor L. Villemagne ${ }^{1,2}$, John Drago ${ }^{3}$, Svetlana Pejoska ${ }^{1}$, Rajinder K. Dhamija ${ }^{4}$, Rachel S. Mulligan ${ }^{1}$, Julia R. Ellis ${ }^{1}$, Uwe Ackermann ${ }^{1}$, Graeme O' Keefe ${ }^{1}$, Gareth Jones ${ }^{1}$, Hank F. Kung ${ }^{5}$, Michael J. Pontecorvo ${ }^{6}$, Daniel Skovronsky ${ }^{6}$, and Christopher C. Rowe ${ }^{1}$

${ }^{1}$ Department of Nuclear Medicine and Centre for PET, Austin Health, Melbourne, Victoria, Australia; ${ }^{2}$ Mental Health Research Institute, University of Melbourne, Melbourne, Victoria, Australia; ${ }^{3}$ Howard Florey Institute, University of Melbourne, and Centre for Neuroscience, University of Melbourne, Melbourne, Victoria, Australia; ${ }^{4}$ Department of Neurology, Austin Health, Melbourne, Victoria, Australia; ${ }^{5}$ Department of Radiology, University of Pennsylvania, Philadelphia, Pennsylvania; and ${ }^{6}$ Avid

Radiopharmaceuticals Inc., Research and Development, Philadelphia, Pennsylvania

PET provides a noninvasive means to evaluate the functional integrity of the presynaptic monoaminergic system in the living human brain. Methods: In this study, a novel ${ }^{18} \mathrm{~F}$-labeled tetrabenazine derivative, ${ }^{18} \mathrm{~F}-(+)$ fluoropropyldihydrotetrabenazine ( $\left.{ }^{18} \mathrm{~F}-\mathrm{AV}-133\right)$, was used for the noninvasive assessment of the vesicular monoamine transporters type 2 (VMAT2) in 17 Parkinson disease (PD) patients and 6 healthy controls. The binding potential (BP) of ${ }^{18} \mathrm{~F}-\mathrm{AV}-133$ was calculated using Logan graphical analysis. Voxel-based and volume-of-interest-based analyses of $\mathrm{BP}$ images were performed to examine brain regional reductions in VMAT2 density in PD. Results: VMAT2 BP was decreased by $81 \%$ in the posterior putamen, $70 \%$ in the anterior putamen, and $48 \%$ in the caudate nucleus of PD patients. Voxelbased analysis demonstrated VMAT2 reductions in the striatum and mid brain of PD patients. Furthermore, VMAT2 BPs in the caudate nuclei significantly correlated with the clinical severity of PD. Conclusion: These findings indicate that the novel ${ }^{18} \mathrm{~F}-$ labeled ligand ${ }^{18} \mathrm{~F}-\mathrm{AV}-133$ can sensitively detect monoaminergic terminal reductions in PD patients. Studies with ${ }^{18} \mathrm{~F}-\mathrm{AV}-133$ may allow the presymptomatic identification of individuals with disorders characterized by degeneration of dopaminergic nigrostriatal afferents.

Key Words: molecular imaging; neurology; PET; Parkinson disease; VMAT2; PET

J Nucl Med 2010; 51:223-228

DOI: 10.2967/jnumed.109.070094

$\mathbf{P}$ arkinson disease (PD) is a common neurodegenerative disorder clinically characterized by resting tremor, rigidity, bradykinesia, and gait disturbance resulting in falls. The extrapyramidal symptoms in PD are caused by the loss of dopamine neurons in the substantia nigra, with consequent

Received Sep. 1, 2009; revision accepted Oct. 30, 2009.

For correspondence or reprints contact: Christopher C. Rowe,

Department of Nuclear Medicine, Centre for PET, Austin Health, 145

Studley Rd., Heidelberg, VIC 3084, Australia.

E-mail: Christopher.Rowe@austin.org.au

COPYRIGHT () 2010 by the Society of Nuclear Medicine, Inc. prominent dopaminergic terminal loss in the striatum. Increasing evidence suggests that the noninvasive evaluation of nigrostriatal dopaminergic integrity by PET and SPECT may provide useful clinical information for the early diagnosis of PD (1-6). Several radiotracers have been proposed for this purpose, including ${ }^{18} \mathrm{~F}$-labeled levodopa $\left({ }^{18} \mathrm{~F}-\mathrm{FDOPA}\right)$, for assessing presynaptic dopamine synthesis; ${ }^{11} \mathrm{C}-\mathrm{WIN}-35,428$ and ${ }^{123} \mathrm{I}-\beta$-CIT, for binding to the dopamine transporter (DAT); and ${ }^{11} \mathrm{C}$-labeled dihydrotetrabenazine $\left({ }^{11} \mathrm{C}\right.$-DTBZ), for binding to the vesicular monoamine transporter type 2 (VMAT2) (1-6). VMAT2 is the transporter responsible for the uptake and storage of dopamine and other monoamines into vesicles in monoamine-containing neurons. VMAT2 is mainly located on synaptic vesicles at the nerve terminals but also on dense core vesicles in nerve cell bodies and dendrites (7). Previous PET studies with ${ }^{11} \mathrm{C}$-DTBZ have shown decreased binding to VMAT2 in the striatum of PD patients $(5,8,9)$. A reduction of VMAT2 reflects the degeneration of nigrostriatal dopaminergic neurons and is less susceptible than DAT to compensatory changes occurring with the loss of dopaminergic neurons $(9,10)$. The in vivo measurement of VMAT2 density could, thus, be useful for the early and differential diagnosis of PD.

A novel ${ }^{18} \mathrm{~F}$-labeled tetrabenazine derivative, ${ }^{18} \mathrm{~F}-(+)$ fluoropropyldihydrotetrabenazine $\left({ }^{18} \mathrm{~F}-\mathrm{AV}-133\right)$, that selectively binds with high affinity to VMAT2 has been developed to assess VMAT2 density in vivo with PET $(11,12)$. To evaluate the utility of this novel tracer for assessing presynaptic neuronal integrity in PD, a PET study was performed to compare ${ }^{18} \mathrm{~F}-\mathrm{AV}-133$ binding in $\mathrm{PD}$ patients and healthy controls (HCs).

\section{MATERIALS AND METHODS}

\section{Subjects}

Written informed consent was obtained from all participants. Approval for the study was obtained from the Austin Health 
Human Research Ethics Committee. HCs were recruited by advertisement in the community, and participants fulfilling clinical criteria for PD (13) were recruited from movement disorder clinics. Seventeen PD subjects aged $67.4 \pm 9.3$ y (mean $\pm \mathrm{SD}$; range, 53-82 y) and 6 age-matched HCs aged $65.2 \pm 4.5$ y (range, $57-70$ y) were included in the study.

All subjects underwent neurologic and neuropsychologic examination. Fifteen patients were receiving carbidopa-levodopa, 1 was receiving selegiline, and 1 was receiving levodopa and benserazide. The neurologic evaluation included the assessment of duration of illness, Hoehn and Yahr score, and motor subscale (section III) of the Unified Parkinson Disease Rating Scale $\left(\mathrm{UPDRS}_{\mathrm{m}}\right)$ while the patient was not taking medication $(24 \mathrm{~h}$ after the last medication dosage). Furthermore, clinical laterality symptoms were assigned scores (Table 1) to allow correlational analysis with the ${ }^{18} \mathrm{~F}-\mathrm{AV}-133$ PET results. Treatment was resumed before the PET scan. The neuropsychologic evaluation included the mini-mental state examination (MMSE), clinical dementia rating (CDR), hospital anxiety and depression scale (HADS), fluctuating assessment scale, logical memory score, and verbal fluency scores.

\section{Radiosynthesis of ${ }^{18} \mathrm{~F}-\mathrm{AV}-133$}

${ }^{18} \mathrm{~F}-\mathrm{AV}-133$ was obtained by nucleophilic substitution of the mesylate precursor AV-244 using previously described methods (12), with slight modifications. AV-244 (2 mg in $1 \mathrm{~mL}$ of 50:50 dimethylsulfoxide:acetonitrile [DMSO:ACN]) was reacted with azeotropically dried ${ }^{18} \mathrm{~F}$ for $10 \mathrm{~min}$ at $90^{\circ} \mathrm{C}$. Unreacted fluoride was eliminated from the crude reaction via tC18 Sep Pak (Waters Corp.) purification. ${ }^{18} \mathrm{~F}-\mathrm{AV} 133$ was eluted off the Sep Pak with ACN (1 mL) and purified via semipreparative high-performance liquid chromatography (Zorbax Eclipse XDB-C18 column; Agilent Technologies) $(5 \mu \mathrm{m}, 9.4 \times 250 \mathrm{~mm}$ eluted with 45:55 ACN:20 $\mathrm{mM}$ ammonium acetate, $4.0 \mathrm{~mL} / \mathrm{min}$ flow rate, $11-\mathrm{min}$ retention time). The ${ }^{18} \mathrm{~F}-\mathrm{AV} 133$ fraction was collected and reformulated for injection using a tC18 Sep Pak. The final dose was filtered through a Millex AV polyvinylidene difluoride $0.22-\mu \mathrm{m}$ filter. The average non-decay-corrected radiochemical yield was $24 \%$ after a synthesis time of $60 \mathrm{~min}$, with a radiochemical purity of greater than $95 \%$ and a specific activity ranging from 44.4 to $207.9 \mathrm{GBq} / \mu \mathrm{mol}$.

\section{Imaging Studies}

Each subject received approximately $250 \mathrm{MBq}$ of ${ }^{18} \mathrm{~F}-\mathrm{AV}-133$ by intravenous injection over $1 \mathrm{~min}$. Imaging was performed with a Phillips Allegro PET camera (spatial resolution, $5.2 \mathrm{~mm}$ in full width at half maximum). A rotation transmission sinogram in 3-dimensional (3D) mode with a single ${ }^{137} \mathrm{Cs}$ point source was acquired before the injection of the radiotracer for attenuationcorrection purposes. An initial 90-min dynamic list-mode emission image was acquired in 3D mode after the injection of ${ }^{18} \mathrm{~F}-\mathrm{AV}-133$. List-mode raw data were sorted offline into $4 \times 30$-s, $9 \times 1$-min, $3 \times 3$-min, $10 \times 5$-min, and $2 \times 10$-min frames. Further images were obtained at 120-140 and 180-200 min after injection. The sorted sinograms were reconstructed using a $3 \mathrm{D}$ row-action maximum-likelihood algorithm.

All subjects received a 3D T1-weighted magnetizationprepared rapid-acquisition gradient-echo MRI scan for screening of other diseases and coregistration with the PET images.

\section{Image Analysis}

The dynamic PET images were converted into binding potential (BP) parametric images through Logan graphical analysis with

\begin{tabular}{lc}
\hline TABLE 1. Clinical Laterality Scores & \\
\hline \multicolumn{1}{c}{ Side } & Score \\
\hline Right-sided symptoms & 2 \\
Right $>$ left & 1 \\
Bilateral & 0 \\
Left $>$ right & -1 \\
Left-sided symptoms & -2 \\
\hline
\end{tabular}

PMOD software (version 3.0; PMOD Technologies), using the primary visual cortex, a region relatively devoid of monoaminergic terminals, as input function (14). Parametric ${ }^{18} \mathrm{~F}-\mathrm{AV}-133 \mathrm{PET} /$ MRI coregistered images were spatially normalized into the Montreal Neurologic Institute MRI brain template standard stereotactic space using statistical parametric mapping software (SPM5; Wellcome Department of Cognitive Neurology). Spheric volumes of interest (VOIs) $(5 \mathrm{~mm}$ in diameter) were placed over a spatially normalized MR image in the caudate nucleus, anterior and posterior putamen, and mid brain. VOIs were then transferred onto BP parametric images, and regional BP values were calculated using PMOD. Asymmetry indices between right (R) and left $(\mathrm{L})$ caudate and putamen were calculated as follows: $(\mathrm{R}-\mathrm{L}) /([\mathrm{R}+\mathrm{L}] / 2)$.

\section{Statistical Analysis}

Regional BP values were statistically compared using the Student $t$ test, and clinical data were statistically compared using the Mann-Whitney $U$ test. Correlations between the ${ }^{18} \mathrm{~F}-\mathrm{AV}-133$ $\mathrm{BP}$ values and clinical data were performed using a nonparametric Spearman rank correlation analysis. Statistical significance for each analysis was defined as a $P$ value less than 0.05 . These analyses were performed using Prism 5 (GraphPad Software). Additionally, SPM5 was used to evaluate intergroup BP differences on a voxelwise basis. Group comparisons between PD patients and HCs were performed by voxel-by-voxel $t$ tests, accepting only voxels surviving false-discovery rate correction for the entire volume at a $P$ value less than 0.05 , to avoid falsepositive results.

\section{RESULTS}

Demographic and clinical data are summarized in Table 2. The PD group contained a higher proportion of men than did the HC group. All patients were considered to have mild to moderate PD (off-medication state Hoehn and Yahr scores, 1-3: stage 1, $n=4$; stage $1.5, n=2$; stage 2, $n=4$; stage $2.5, n=2$; and stage $3, n=1$ patients). The UPDRS $\mathrm{Um}_{\mathrm{m}}$ in the off-medication state and HADS were significantly higher in PD patients than in HCs. There was no difference between the groups in CDR, fluctuating assessment scale, logical memory score, and verbal fluency score. The Hoehn and Yahr and UPDRS scores in the off-medication state were unavailable in 4 PD patients.

Lower ${ }^{18} \mathrm{~F}-\mathrm{AV}-133$ BPs were observed in the putamen, caudate, and mid brain of PD patients. Representative ${ }^{18} \mathrm{~F}-$ AV-133 BP PET images in a healthy elderly control (66-yold man) and a PD patient (61-y-old man; Hoehn and Yahr score, 2) are shown in Figure 1A. Voxel-based group comparison of ${ }^{18} \mathrm{~F}-\mathrm{AV}-133 \mathrm{BP}$ images showed significantly 

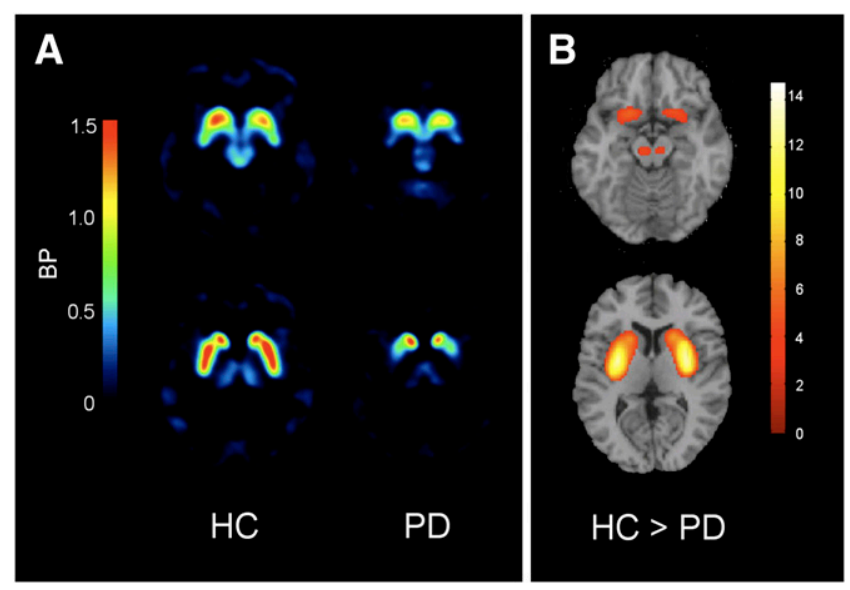

FIGURE 1. (A) Representative images of ${ }^{18} \mathrm{~F}-\mathrm{AV}-133$ PET $\mathrm{BP}$ in $\mathrm{HC}$ and $\mathrm{PD}$ patient. (B) Areas of reduction in $\mathrm{BP}$ of $\mathrm{PD}$ patients, compared with $\mathrm{HCs}$, in SPM analysis. Color bars represent $t$ values. $P<0.05$, corrected for multiple comparisons.

lower BP in PD patients than in HCs in the striatum (L: $-30,-4,2, x, y, z ; \mathrm{Z}=7.07 ; \mathrm{R}: 32,-4,8, x, y, z ; \mathrm{Z}=$ 6.69) and substantia nigra ( $\mathrm{L}:-6,-20,-14, x, y, z ; \mathrm{Z}=$ 3.67; R: 10, $-18,-14, x, y, z ; Z=3.45$ ) (Fig. 1B). In contrast, no brain region in PD patients showed significantly higher ${ }^{18} \mathrm{~F}-\mathrm{AV}-133 \mathrm{BP}$ than that in the brain region of HCs. VOI-based analysis similarly indicated a significantly lower BP in the caudate nucleus, putamen, and mid brain of
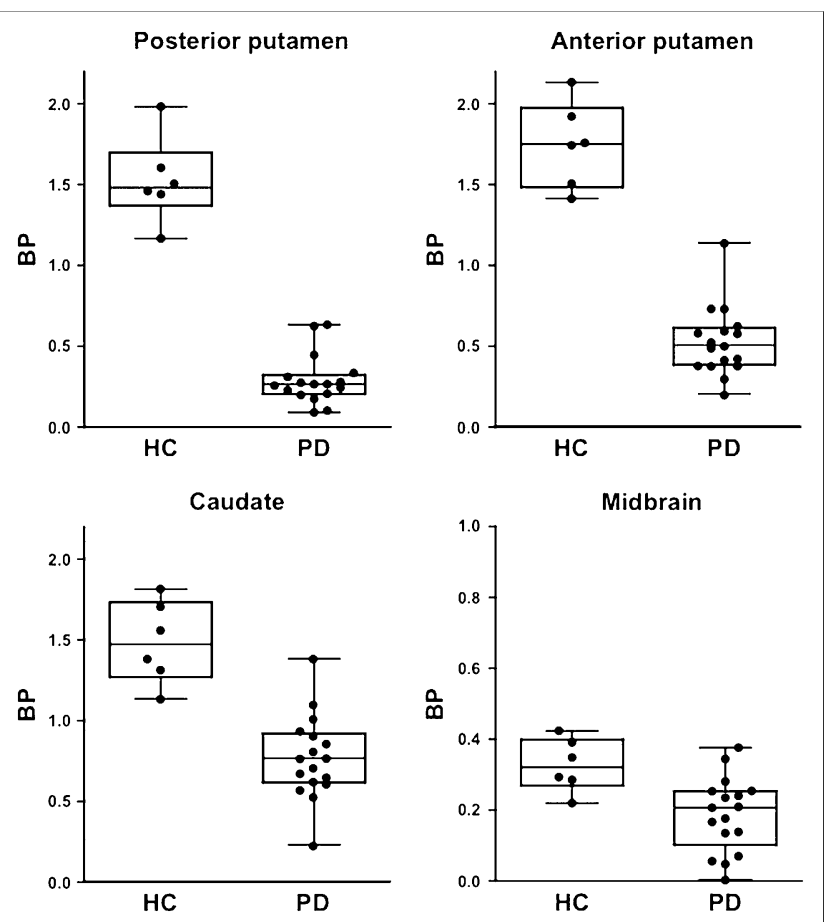

FIGURE 2. ${ }^{18} \mathrm{~F}-\mathrm{AV}-133 \mathrm{PET}$ BP from VOI analysis in HCs and PD patients.
TABLE 2. Demographic Characteristics of Subjects

\begin{tabular}{|c|c|c|}
\hline Characteristic & $\mathrm{HC}$ & PD \\
\hline$n$ & 6 & 17 \\
\hline Mean age $\pm S D(y)$ & $65.2 \pm 4.5$ & $67.4 \pm 9.3$ \\
\hline $\operatorname{Sex}(M / F)$ & $2 / 4$ & $17 / 0$ \\
\hline MMSE & $30.0 \pm 0.0$ & $28.9 \pm 1.2$ \\
\hline CDR & 0.0 & $0.2 \pm 0.5$ \\
\hline Symptom onset (y) & & $7.6 \pm 5.5$ \\
\hline Disease duration $(\mathrm{y})$ & & $5.6 \pm 5.0$ \\
\hline UPDRS $_{\mathrm{m}}$ & 0.0 & $20.6 \pm 9.9^{*}$ \\
\hline \multicolumn{3}{|l|}{ UPDRS $_{m}$ subscales } \\
\hline Bradykinesia & 0.0 & $2.0 \pm 0.9^{*}$ \\
\hline Rigidity & 0.0 & $1.8 \pm 0.7^{\star}$ \\
\hline Fluctuating Assessment Scale & 0.0 & $0.9 \pm 1.7$ \\
\hline Logical memory & $14.5 \pm 5.2$ & $11.7 \pm 4.1$ \\
\hline Verbal fluency & $45.5 \pm 9.9$ & $32.7 \pm 10.6$ \\
\hline HADS & $6.3 \pm 4.8$ & $11.2 \pm 4.9^{*}$ \\
\hline
\end{tabular}

PD patients (Fig. 2; Table 3). No overlap was observed between the ${ }^{18} \mathrm{~F}-\mathrm{AV}-133 \mathrm{BP}$ values in the putamen of PD patients and HCs. The greatest BP decline in PD was observed in the posterior putamen $(-81 \%)$, followed by the anterior putamen $(-70 \%)$, caudate $(-48 \%)$, and mid brain $(-43 \%)$. In the posterior putamen, ${ }^{18} \mathrm{~F}-\mathrm{AV}-133 \mathrm{BP}$ values in all PD patients were more than 3 SDs below those in HCs.

The correlation between regional BP values and UPDRS $_{\mathrm{m}}$ and HADS scores was also examined. As shown in Figure 3, UPDRS $\mathrm{m}_{\mathrm{m}}$ bradykinesia subscores in PD patients negatively correlated with ${ }^{18} \mathrm{~F}-\mathrm{AV}-133 \mathrm{BP}$ values in the caudate $(r=-0.59, P=0.02)$, and a trend was observed in the anterior putamen $(r=-0.50, P=0.06)$. However, no significant correlations were found in the posterior putamen or when the $\mathrm{UPDRS}_{\mathrm{m}}$ rigidity subscores were examined. No significant correlation was observed between regional BP values and the HADS scores. Conversely, there was a significant inverse correlation between ${ }^{18} \mathrm{~F}-\mathrm{AV}-133$ asymmetry indices in the striatum and the clinical laterality scores (Fig. 3).

\begin{tabular}{lcc}
\hline \multicolumn{2}{c}{ TABLE 3. BPs of ${ }^{18} \mathrm{~F}-\mathrm{AV}-133 \mathrm{PET}$} \\
Area & $\mathrm{HC}$ & $\mathrm{PD}$ \\
Caudate nuclei & $1.48 \pm 0.26$ & $0.77 \pm 0.26^{\star}$ \\
Right caudate nuclei & $1.47 \pm 0.25$ & $0.79 \pm 0.27^{\star}$ \\
Left caudate nuclei & $1.50 \pm 0.26$ & $0.75 \pm 0.27^{\star}$ \\
Anterior putamen & $1.75 \pm 0.26$ & $0.53 \pm 0.21^{\star}$ \\
Right anterior putamen & $1.78 \pm 0.26$ & $0.56 \pm 0.26^{\star}$ \\
Left anterior putamen & $1.72 \pm 0.28$ & $0.49 \pm 0.21^{\star}$ \\
Posterior putamen & $1.53 \pm 0.27$ & $0.29 \pm 0.15^{\star}$ \\
Right posterior putamen & $1.56 \pm 0.26$ & $0.32 \pm 0.21^{\star}$ \\
Left posterior putamen & $1.49 \pm 0.27$ & $0.26 \pm 0.12^{\star}$ \\
Mid brain & $0.33 \pm 0.08$ & $0.19 \pm 0.10^{\star}$
\end{tabular}

*Significantly different from $\mathrm{HC}(P<0.05)$. 


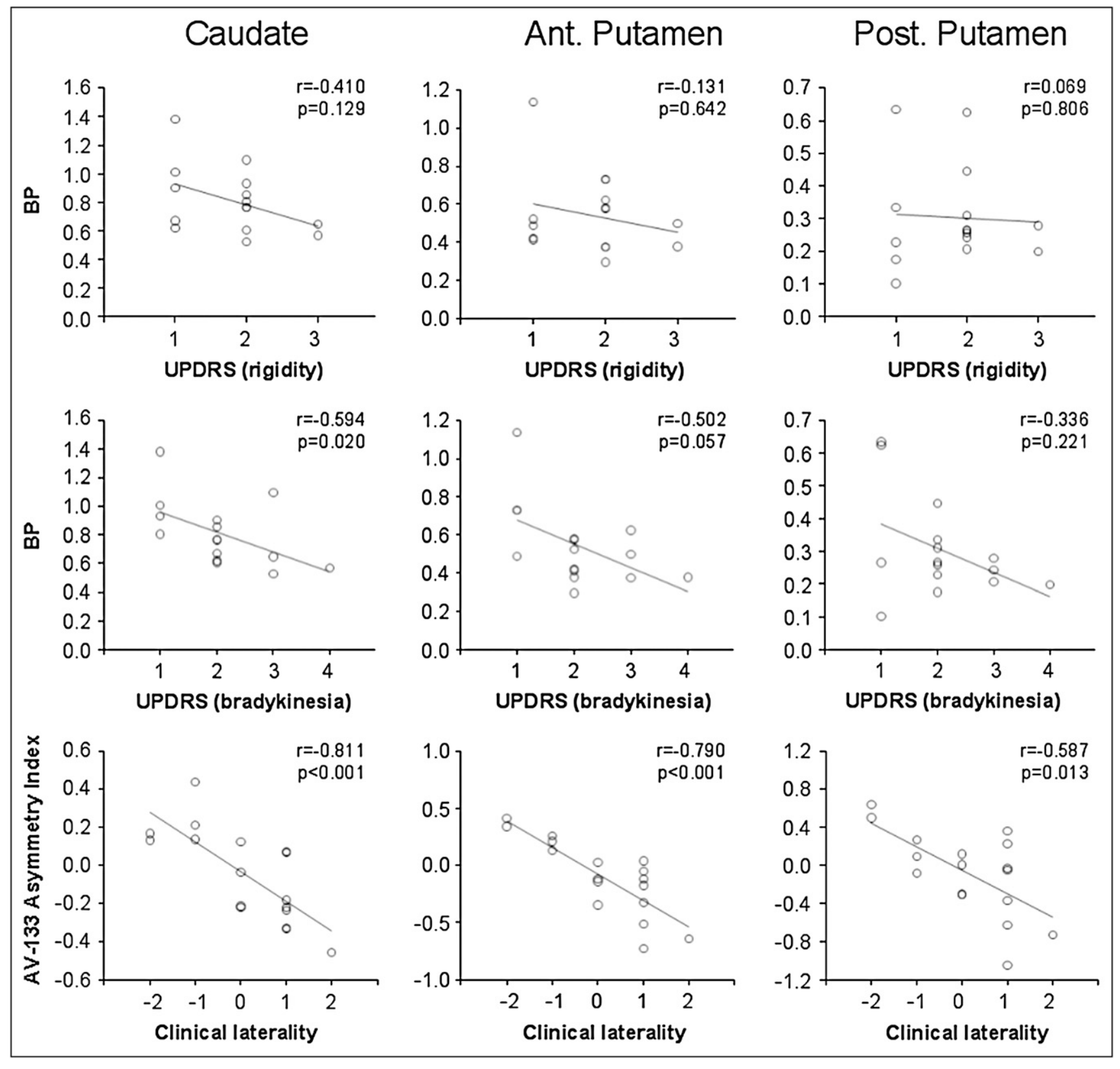

FIGURE 3. Correlation of rigidity and bradykinesia subscores of UPDRS $m$ and clinical laterality ratings with ${ }^{18} \mathrm{~F}-\mathrm{AV}-133 \mathrm{PET}$ $\mathrm{BP}$ in striatum. Although there was strong association between asymmetry indices and laterality scores, only VMAT2 BP in caudate correlated with bradykinesia UPDRS $_{\mathrm{m}}$ subscores. Ant. = anterior; Post. = posterior.

\section{DISCUSSION}

Both DAT and VMAT2 have been proposed as markers of dopaminergic nerve terminal integrity. Imaging with DAT-specific radioligands has been reported to be a sensitive index of presynaptic neuronal degeneration, enabling the early and accurate diagnosis of patients with suspected PD (15). However, DAT is susceptible to dopaminergic regulation and can be altered by treatment with L-DOPA, deprenyl, cocaine, and amphetamine (16). In contrast, whereas VMAT2 is common to all monoaminergic neurons and not just dopaminergic ones, it is less susceptible to the effects of L-DOPA or other dopaminergic drugs (16-18). Thus, VMAT2 imaging may be a more reliable marker to monitor dopamine terminal integrity in PD patients. Currently, ${ }^{11} \mathrm{C}$-DTBZ is the only available PET tracer for the noninvasive quantification of VMAT2 density in the brain. Unfortunately, the 20-min radioactive decay half-life of ${ }^{11} \mathrm{C}$ limits the use of ${ }^{11} \mathrm{C}$-DTBZ to centers with an on-site cyclotron and ${ }^{11} \mathrm{C}$ radiochemistry expertise. Consequently, access to ${ }^{11} \mathrm{C}$-DTBZ is restricted, and the high cost of studies is prohibitive for routine clinical use. To overcome these limitations, a tracer for imaging VMAT2 that can be 
labeled with ${ }^{18} \mathrm{~F}$ is required. The 110 -min radioactive decay half-life of ${ }^{18} \mathrm{~F}$ permits the centralized production and regional distribution as currently practiced worldwide in the supply of ${ }^{18}$ F-FDG for clinical use.

The current PET study showed significantly lower VMAT2 densities as measured by ${ }^{18} \mathrm{~F}-\mathrm{AV}-133$ in the putamen, caudate, and substantia nigra of PD patients. The greatest VMAT2 reductions were observed in the posterior putamen $(-81 \%)$, followed by the anterior putamen $(-70 \%)$, and caudate nucleus $(-48 \%)$. These results are consistent with previous ${ }^{11} \mathrm{C}$-DTBZ PET results showing the highest reduction $(71 \%-77 \%)$ in the posterior putamen of PD patients $(8,9,19,20)$. Our observations are also consistent with postmortem reports showing $88 \%$ reduction of VMAT2 immunoreactivity in the putamen of PD patients (21). There was no intergroup overlap between the putaminal BP of PD patients and controls. Actually, BP values in the posterior putamen of all PD patients were more than 3 SDs below those of HCs. These results suggest that ${ }^{18} \mathrm{~F}-\mathrm{AV}-133$ is a highly sensitive tool to detect early dopaminergic dysfunction in PD patients. A longitudinal study in mildly affected PD patients or individuals at risk of PD will elucidate the usefulness of this tracer for early or presymptomatic diagnosis of PD (6). As shown in previous PET and SPECT studies $(19,22,23)$, the evaluation of presynaptic dopaminergic integrity would also be useful in the differential diagnosis of dementia with Lewy bodies (DLB) from Alzheimer disease (AD), for which severe nigrostriatal dopaminergic degeneration, similar to that observed in PD, is present in DLB but not in AD. Additional studies comparing ${ }^{18} \mathrm{~F}-\mathrm{AV}-133$ binding in DLB and AD patients are warranted. Likewise, VMAT2 imaging could be applied to evaluate cases of familial PD for the detection of nigrostriatal degeneration at early, presymptomatic stages and the assessment of subjects with rapid eye movement sleep behavior disorder and also to evaluate subjects considered to have mild cognitive impairment who might present with mild or inconclusive motor symptoms suggestive of a pre-DLB stage.

Dopamine is released not only from axon terminals in the striatum but also from the cell bodies and dendrites in the substantia nigra. Consistently, VMAT2 is also localized in extrastriatal regions including the substantia nigra (7). Human PET studies using stereoisomers of ${ }^{11} \mathrm{C}-\mathrm{DTBZ}$ demonstrated specific in vivo binding of ${ }^{11} \mathrm{C}-\mathrm{DTBZ}$ in the mid brain (24), and in vivo studies in mice showed ${ }^{18} \mathrm{~F}$ AV-133 binding to VMAT2 in the substantia nigra (11). Previous ${ }^{11} \mathrm{C}$-DTBZ PET studies demonstrated a $50 \%$ VMAT2 reduction in the substantia nigra of PD patients (20). In a similar fashion, the present study demonstrated a $43 \%$ reduction of ${ }^{18} \mathrm{~F}-\mathrm{AV}-133$ binding in the substantia nigra of PD patients, consistent with previous reports of VMAT2 reduction in the PD brain $(20,24)$.

Significant correlations between clinical severity PD scores and ${ }^{18} \mathrm{~F}-\mathrm{AV}-133$ binding were observed in the caudate nucleus but not in the putamen. This result conflicts with previous PET reports showing correlation of UPDRS score with ${ }^{11} \mathrm{C}$-DTBZ binding or ${ }^{18} \mathrm{~F}$-FDOPA uptake in the putamen $(9,25)$. The lack of correlation in the putamen might be caused by the limited sample size or, more likely, a floor effect because the putamen is more severely affected than the caudate nucleus.

Depression is one of the most common complications in PD, contributing to poor quality of life and disability. HADS scores, mainly driven by the depression scales, were significantly higher in PD patients. A VMAT2 inhibitor, reserpine, is known to induce depressionlike symptoms in humans. Although a previous study demonstrated the association of lower striatal VMAT2 density and cocaineinduced mood disorders (26), we did not find a correlation between HADS scores and ${ }^{18} \mathrm{~F}-\mathrm{AV}-133$ binding in PD patients, suggesting a weak association between striatal VMAT2 density and mood function in PD patients. A strong inverse correlation was found between ${ }^{18} \mathrm{~F}-\mathrm{AV}-133$ asymmetry indices and clinical laterality scores, indicating that ${ }^{18} \mathrm{~F}-\mathrm{AV}-133$ can correctly identify the most affected side. Additional studies using larger sample populations and less affected individuals are required to more fully examine the correlation of regional ${ }^{18} \mathrm{~F}-\mathrm{AV}-133$ binding with clinical severity of PD.

\section{CONCLUSION}

Significant VMAT2 reductions were successfully detected in the striatum and mid brain of PD patients with ${ }^{18} \mathrm{~F}-\mathrm{AV}-133$. These observations indicate that ${ }^{18} \mathrm{~F}-\mathrm{AV}-133$ is a suitable ligand for the noninvasive assessment of VMAT2 density in the human brain.

\section{ACKNOWLEDGMENTS}

We thank Drs. Sylvia Gong, Henry Tochon-Danguy, and Andrew Hughes for their crucial role in patient recruitment and PET examinations, and Tania Petts, Tim Saunder, and Jason Bradley for their role during PET examinations and image processing. This study was supported in part by funds from Avid Radiopharmaceuticals Inc. and the Austin Hospital Medical Research Foundation.

\section{REFERENCES}

1. Ravina B, Eidelberg D, Ahlskog JE, et al. The role of radiotracer imaging in Parkinson disease. Neurology. 2005;64:208-215.

2. Wong DF, Yung B, Dannals RF, et al. In vivo imaging of baboon and human dopamine transporters by positron emission tomography using $\left[{ }^{11} \mathrm{C}\right] \mathrm{WIN}$ 35,428. Synapse. 1993;15:130-142.

3. Villemagne V, Yuan J, Wong DF, et al. Brain dopamine neurotoxicity in baboons treated with doses of methamphetamine comparable to those recreationally abused by humans: evidence from $\left[{ }^{11} \mathrm{C}\right] \mathrm{WIN}-35,428$ positron emission tomography studies and direct in vitro determinations. J Neurosci. 1998;18: 419-427.

4. Pirker W, Djamshidian S, Asenbaum S, et al. Progression of dopaminergic degeneration in Parkinson's disease and atypical parkinsonism: a longitudinal $\beta$-CIT SPECT study. Mov Disord. 2002;17:45-53.

5. Frey KA, Koeppe RA, Kilbourn MR, et al. Presynaptic monoaminergic vesicles in Parkinson's disease and normal aging. Ann Neurol. 1996;40:873-884.

6. Stoessl AJ. Positron emission tomography in premotor Parkinson's disease. Parkinsonism Relat Disord. 2007;13(suppl 3):S421-S424. 
7. Nirenberg MJ, Chan J, Liu Y, Edwards RH, Pickel VM. Ultrastructural localization of the vesicular monoamine transporter-2 in midbrain dopaminergic neurons: potential sites for somatodendritic storage and release of dopamine. J Neurosci. 1996;16:4135-4145.

8. Lee CS, Samii A, Sossi V, et al. In vivo positron emission tomographic evidence for compensatory changes in presynaptic dopaminergic nerve terminals in Parkinson's disease. Ann Neurol. 2000;47:493-503.

9. Martin WR, Wieler M, Stoessl AJ, Schulzer M. Dihydrotetrabenazine positron emission tomography imaging in early, untreated Parkinson's disease. Ann Neurol. 2008;63:388-394.

10. Chen MK, Kuwabara H, Zhou Y, et al. VMAT2 and dopamine neuron loss in a primate model of Parkinson's disease. J Neurochem. 2008;105:78-90.

11. Kung MP, Hou C, Goswami R, Ponde DE, Kilbourn MR, Kung HF. Characterization of optically resolved 9-fluoropropyl-dihydrotetrabenazine as a potential PET imaging agent targeting vesicular monoamine transporters. Nucl Med Biol. 2007;34:239-246.

12. Goswami R, Ponde DE, Kung MP, Hou C, Kilbourn MR, Kung HF. Fluoroalkyl derivatives of dihydrotetrabenazine as positron emission tomography imaging agents targeting vesicular monoamine transporters. Nucl Med Biol. 2006;33: 685-694.

13. Calne DB, Snow BJ, Lee C. Criteria for diagnosing Parkinson's disease. Ann Neurol. 1992;32(suppl):S125-S127.

14. Logan J, Fowler JS, Volkow ND, Wang GJ, Ding YS, Alexoff DL. Distribution volume ratios without blood sampling from graphical analysis of PET data. J Cereb Blood Flow Metab. 1996;16:834-840.

15. Marshall V, Grosset D. Role of dopamine transporter imaging in routine clinical practice. Mov Disord. 2003;18:1415-1423.

16. Wilson JM, Kish SJ. The vesicular monoamine transporter, in contrast to the dopamine transporter, is not altered by chronic cocaine self-administration in the rat. J Neurosci. 1996;16:3507-3510.
17. Kilbourn MR, Frey KA, Vander Borght T, Sherman PS. Effects of dopaminergic drug treatments on in vivo radioligand binding to brain vesicular monoamine transporters. Nucl Med Biol. 1996;23:467-471.

18. Wilson JM, Levey AI, Rajput A, et al. Differential changes in neurochemical markers of striatal dopamine nerve terminals in idiopathic Parkinson's disease. Neurology. 1996;47:718-726.

19. Gilman S, Koeppe RA, Little R, et al. Striatal monoamine terminals in Lewy body dementia and Alzheimer's disease. Ann Neurol. 2004;55:774-780.

20. Bohnen NI, Albin RL, Koeppe RA, et al. Positron emission tomography of monoaminergic vesicular binding in aging and Parkinson disease. J Cereb Blood Flow Metab. 2006;26:1198-1212.

21. Miller GW, Erickson JD, Perez JT, et al. Immunochemical analysis of vesicular monoamine transporter (VMAT2) protein in Parkinson's disease. Exp Neurol. 1999; 156:138-148.

22. Hu XS, Okamura N, Arai H, et al. ${ }^{18} \mathrm{~F}$-fluorodopa PET study of striatal dopamine uptake in the diagnosis of dementia with Lewy bodies. Neurology. 2000;55: 1575-1577.

23. McKeith I, O'Brien J, Walker Z, et al. Sensitivity and specificity of dopamine transporter imaging with ${ }^{123} \mathrm{I}-\mathrm{FP}-\mathrm{CIT}$ SPECT in dementia with Lewy bodies: a phase III, multicentre study. Lancet Neurol. 2007;6:305-313.

24. Koeppe RA, Frey KA, Kuhl DE, Kilbourn MR. Assessment of extrastriatal vesicular monoamine transporter binding site density using stereoisomers of $\left[{ }^{11} \mathrm{C}\right]$ dihydrotetrabenazine. J Cereb Blood Flow Metab. 1999;19: 1376-1384.

25. Dhawan V, Ma Y, Pillai V, et al. Comparative analysis of striatal FDOPA uptake in Parkinson's disease: ratio method versus graphical approach. J Nucl Med. 2002;43:1324-1330.

26. Little KY, Krolewski DM, Zhang L, Cassin BJ. Loss of striatal vesicular monoamine transporter protein (VMAT2) in human cocaine users. Am J Psychiatry. 2003;160:47-55. 